\title{
A brief analysis of the existing problems of College Students' autonomous learning in English

\author{
Maohua SUN
}

Foreign Language Department, Dalian Jiao Tong University, Liaoning Province, China

Isummer817@163.com

Keywords: college students; autonomous learning in English; influential factors.

Abstract. in this paper, the factors that affect college students' English autonomous learning are divided into two categories: individual factors and common factors, and a specific analysis is made. The author believes that the analysis of these causes. It can help college students to learn English autonomously, and then promote their self-learning ability and self-learning habits.

\section{浅析大学生英语自主学习存在的问题}

\author{
孙茂华 \\ 大连交通大学, 大连, 辽宁省, 中国 \\ summer_817@163.com
}

关键词：大学生;英语自主学习;影响因素

中文摘要. 本文将影响大学生英语自主学习的因素分为个性因素和共性因素两类, 并进行了 具体分析。作者认为, 分析这些因素, 可以为大学生的英语自主学习提供帮助, 进而促进其自 主学习能力的提高和自主学习习惯的养成。

\section{1. 自主学习的实质}

自主学习的核心特征是自觉性。目前我国的报刊或著作中论述了许多自主学习的特征或 特点, 不论有没有提到自觉性, 但往往论述的含义是自觉性的特点。自觉性是人类四大意志 品质之一, 又叫目的性和独立性, 是指人们行动的目的和动机, 或对感情和行为的支配过程 中, 不屈服于外人的压力, 而根据自己的认识、情感、态度或价值观, 独立自主决定并付诸 行动。我们在调查中看到, 在学校里学习成绩优秀的学生, 多半是自觉的学习。他们自己确 定学习的目的（或有着自己的学习动机）, 在学习过程中能自我选择学习内容和选定适合自 己的学习方法, 擅长自我解决学习中的问题并能不断地克服学习的困难, 能自我评估 (或评 价) 学习的结果。伴随这种自觉学习, 必然产生自我调控的学习, 这就是学习中的反思性或 自我监控性, 心理学中称其为 “元认知”。它包括元认知知识和元认知监控两大部分（董奇, 1989）。学生学习的元认知知识主要包括: 关于所学教材特点的知识（例如教材的长短、难 度、熟悉性、趣味性、结构性) 、关于学习任务方面的知识 (例如对学生提出知识特点的记 忆要求、提出要求以及评价等）、关于策略方面的知识（例如各种学习活动与方式的特点, 调节学习过程的方法等) 、关于学生本人特点方面的知识（例如自己的兴趣、爱好、能力、 情感、态度等）。而学生学习的元认知监控就是在实际学习过程中如何有效地、适当地协调 上述元认知知识的四个方面的相互作用。具体地说, 就是学生根据教学内容特点、自身特点、 问题要求等相应地做出计划, 选择合适而有效的策略, 评价每一操作的有效性, 检查结果, 修正策略, 并对再次出现的问题采取有效的补救措施等。自主学习的构成因素。关于自主学 
习有哪些因素构成? 其内在结构是什么? 不同研究者和理论之间存在差异。美国科罗拉多大 学的研究者 Traci Sitamann (2011) 及其同事在对自主学习相关主要理论分析基础上提出的自 主学习的内在结构对于我们认识这个问题具有重要意义。他们首先对自我效能理论

（self-efficacy theory）、控制理论 (control theory)、行动调节理论(action regulation theory)、 资源分配理论( resource allocation theory) 以及Zimmerman从教育心理学角度提出的自我调节 学习理论 (Self-regulated learning) 等进行综合分析基础上提出, 自主学习内在结构包括 3 个 方面, 共16个核心成分。第一个方面为监管动因（regulatory agents），包括1个核心成分，即 学习者自觉设置的学习目标水平。学习目标水平对于整个自主学习发挥着指引作用, 并作为 学习过程中不断自我监控和调整的根本依据; 第二个方面为监管机制 (regulator mechanisms), 它决定着学习者能否以有效和组织化的方式实现之前自觉设置的目标, 它是自主学习能否成 功的关键, 包括12个核心成分: 计划 (planning)、监控 (monitoring)、元认知 (meta cognition)、 注意（attention）、学习策略（Learning strategies）、坚持性（persistence）、时间管理（time management）、环境组织（environmental structuring）、帮助寻求（help seeking）、动机

(motivation)、情绪控制 (emotion control) 和努力 (effort); 第三个方面为监管评价 (regulatory appraisal）, 它负责评价整个目标的实现进度, 并决定着学习者是否继续向着之前确定的目标 前进，包括3个核心成分：自我评价（self-evaluation）、归因（attributions）和自我效能

（self-efficacy）。Traci Sitamann及其同事提出的自主学习的结构, 突出了自主学习的自觉性 特征, 是目前为止看到的关于自主学习构成因素的理论中最为全面的一个, 但是由于该结构 主要是在对以往研究和理论整合分析的基础上产生的, 因此其一些成分之间存在交叉重叠, 需要在未来研究中进行调整。因此, 运用自主学习方式, 不仅可以提高学生学习成绩, 而且 对自我定向、自强独立、自我管理等在终身发展中具有重要作用的能力的提高都有重要意义。 这也是古人所说的 “君子深造以道, 欲其自得之也” 的道理。

\section{2. 英语自主学习的内涵及影响因素}

自主学习这一概念最早由Holec引入英语教学中。关于英语自主学习的内涵主要有 “能力 说”、“学习过程说” 和 “学习态度说”。“能力说” 是指学习者进行批判性反思、作出决 策以及采取独立行动的能力; “学习过程说” 是指学习者根据制定的学习目标积极主动地监 控和调节个人认知、动机和行为并付出努力的过程; 而 “学习态度说” 则是指学习者愿意而 且确实要对自己的学习行为负责的态度。无论自主学习被看作是一种学习能力、学习过程或 是学习态度, 都需要学习者对自己的学习负责, 这是学习的前提。学习这种行为既需要学习 者的这种学习态度, 也需要学习者安排自己学习过程的能力, 所以自主学习应该更加全面地 被看作是学习者本着对自己学习负责的态度, 自觉主动地调控自己学习过程的一种能力。

学生只有具备了自主学习的能力, 才能在语言学习过程中自主地确立学习目标、制定学 习计划、监控学习过程和评价学习效果, 也才能更好地适应信息化社会, 更好地发掘自己的 个人潜能, 为自己的终身发展奠定基础。然而, 自主学习能力的发展和提高受许多因素的影 响, 既包括来自学习者个体内部因素的影响, 如学习者的个性、学习信念、学习兴趣、自我 效能感等, 也包括来自学习环境、物质条件和文化因素等外部因素的影响。环境是学生取得 自主学习成功的决定性因素之一, 而课堂环境作为学生重要的学习环境对学生的自主学习有 着直接的影响作用, 因此, 在英语教学中, 教师必须关注课堂环境为学生自主学习所提供的 社会和心理支持。

\section{3. 学习资源与环境}

学习者自由选择的程度取决于自主学习的条件, 即自主学习的资源与环境。这就要求学 校、家庭以及教师、家长要给学习者提供充分的可供选择的学习资源, 创设有利于其自主学 
习的环境。从学校的角度来说, 学生学习的场所不局限于教室, 图书馆、阅览室、网络教室、 宿舍等都可以成为其学习的场所, 这就要求学校要为学生提供尽可能多的学习场所并配套相 应的设施，不断完善信息资源库，使学生可以选择适宜的学习场所、借阅到所需要的图书资 料。国外的一些大学还建立了专门的自主学习中心 (self-access centers), 为学生提供咨询服务, 使师生、学生之间的相互交流有了相对稳定的场所, 满足学生自主学习的需要。大量的研究 结果表明: 家庭是学生获得自主学习动机和能力的一个重要的来源, 它在个体形成自主学习 习惯的过程中起着不可或缺的作用。尽管大学生多数的学习行为发生在学校, 但父母的教育 和熏陶对其所产生的影响是深远的。学生自主学习能力的获得是外部调节的整合和内化的结 果, 父母是学生人际环境中的重要因素, 他们对儿童的自律持何种态度将会影响学生的自主 学习水平 (Grolnick \&Ryan, 1989)。父母对孩子的示范、鼓励, 为他们提供学习条件, 能够明 显改善学生的自主学习水平, 并通过学生自我调节能力的改善, 提高他们的学业成绩。对学 习者来说, 他们要善于和充分利用已有的学习资源和环境, 不断提高自己的学习效率和自主 学习能力。以上分析了影响大学生英语自主学习的若干因素, 尽管它们都对大学生的英语自 主学习有影响, 但它们所产生的影响不是孤立的, 一种因素对自主学习的影响往往以另一种 因素为中介，而且它们与学习行为之间的关系往往是双向的。

\section{4. 学习同伴的影响}

自主学习不但是个体的也是社会的, 自主学习不会发生在真空中, 也并非指完全独立的 学习, 同伴间的协商、合作能极大促进个体的自主学习。庞维国博士在总结国内外相关研究 成果的基础上，提出同伴至少在以下三个方而影响学生的自主学习:

\section{1、同伴的自主学习行为和学习成绩影响学生对自身自主学习能力的评估}

个体自身自主学习能力的评估主要借助内部比较和社会性比较: 内部比较指个体把自己 在一个学科领域的成绩与另一个学科领域的成绩进行比较, 进而推断自己的学习能力; 外部比 较指个体拿自己的能力与同辈群体在该领域所取得的成绩进行比较。詹尼斯 (Janice, 2000) 研究了 297 名学生的自主学习能力知觉, 结果发现: 无论男生还是女生, 其自主学习能力知觉 的产生主要的是受社会性比较的影响。这说明个体在评估自己的自主学习能力时, 主要以同 辈群体的能力和成就为参照。

\section{2、同伴的自主学习对学生会产生榜样示范作用}

社会认知理论认为, 自主学习能力的发展是个体把外部学习技能内化为自己能力的过程, 要先后经历观察、模仿、自我控制、自我调节四个阶段 (Schunk,1994)。由此看来, 个体的自 主学习能力首先起源于对榜样的学习观念, 榜样的示范起着极为重要的作用。与成人相比, 同伴之间具有更多的相似之处, 同伴中成功的自主学习者更有可能成为大家模仿的对象。

\section{3、同伴关系影响学生的学业求助}

过去人们一度把学业求助看成是能力不足、依赖、不成熟的表现, 但近年来研究者日益 认识到学习求助具有积极的意义, 它不仅是重要的社会互动过程, 而且是重要的适应性自主 学习策略。在同伴群体中, 最为主要的关系表现为吸引与排斥、合作与竞争。一般说来, 在 相互亲近、情感和谐、团结互助的同伴关系中, 学生的竞争程度下降, 自尊心得到保护和尊 重, 学生之间的地位相对平等, 学业求助所付出的代价较小, 学生之间往往有更多的学业求 助。 


\section{References}

[1] Di ckinsom .Aspects of autonomous learning[ J] .ELT Journal , 1993 ,47/ 4:330 -336 .

[2] Cheng Xiao Tang. On Autonomous Learning [J]. Science education, 1999, (9): 32-39.

[3] Qi Hongbo. The theoretical origin of learner autonomy [J]. Shandong foreign language teaching, 2002, (5): 83-86.

[4] Weiguo pang of autonomous learning and learning and teaching principle and strategy [M]. Shanghai: East China Normal University press, 2003.

[5] Zhang Lixin, Li Xiaoxiang. The comparative study of autonomous learning ability of Chinese Western European students [J]. foreign language community, 2004, (4): 15-23.

[6] Xu Jinfen, accounting for a small sea. Domestic and international review of research on learner autonomy in foreign language circles. [J], 2004, 2 (4): -9.

[7] Dong Qi. (1989). On metacognition. Journal of Beijing Normal University (SOCIAL SCIENCE EDITION).1, 68 - 74.

[8] Govan. (2002). Total order.IN: Winter University Belt cognition and technology group, Wang Wenjing, Qiao Liansheng translation, American curriculum and Education Case Perspective -Justin series. Shanghai: East China Normal University press.

[9] Lin Chong de. (2003). Learning and development - the development and cultivation of the psychological ability of primary and secondary schools. Beijing: Beijing Normal University press.

[10]Pang Weiguo. (2000) some progress since.90 foreign independent study psychology. 4, 12 16. 